\title{
PReS-FINAL-2321: Clinical manifestations of granulomatosis with polyangiitis in 8 children from south-east region of Poland
}

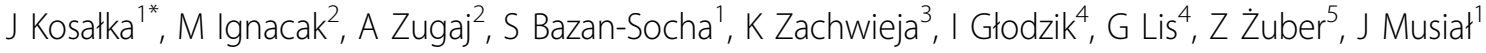 \\ From 20th Pediatric Rheumatology European Society (PReS) Congress \\ Ljubljana, Slovenia. 25-29 September 2013
}

\section{Introduction}

Granulomatosis with polyangiitis (GPA) is a necrotising granulomatous vasculitis affecting the small and medium blood vessels in particular of airways and kidneys. The incidence of GPA in Europe is 25-150 per 1 million per year. This disease typically occurs in the 4 th or 5 th decades of life and in children it usually cause diagnostic and therapeutic difficulties. Subglottic stenosis and nasal deformity are frequently registered in this group of young patients.

\section{Objectives}

The purpose of this study was to analyse the incidence of GPA in large group of children hospitalized in three paediatric reference centres in south-east administrative region of Poland (3,3 million inhabitants), in the years 1995-2013, as well as to investigate their symptoms, laboratory findings and disease outcome.

\section{Methods}

Retrospective study, examining the medical records. Patients with confirmed diagnosis of GPA must meet criteria of American College of Rheumatology and EULAR/PRINTO/PRES for classification of GPA. All patients were subjected to clinical, laboratory, radiology, immunology assessment.

\section{Results}

We found only 8 children with convinced diagnosis of GPA (6 girls, 2 boys). The average age of onset was approx. 11 years (range: 8-16 years), but the average diagnosis delay was approx. 22 months (range: 0-7 years).
The most common clinical features at presentation were constitutional symptoms - weight loss, fever and arthralgia $(87.5 \%-7 / 8)$. The frequency of system involvement at presentation was: kidneys $87.5 \%$ (7/8), lungs 75\% (6/8), ear/nose/sinuses/throat 50\% (4/8), gastrointestinal tract $50 \%(4 / 8)$, skin $37.5 \%(3 / 8)$, eyes $12.5 \%(1 / 8)$, joints $12.5 \%$ $(1 / 8)$ and nervous system $12.5 \%(1 / 8)$. ANCA were positive in all patients. Treatment included: glucocorticosteroids $100 \%$, cyclophosphamide $100 \%$, mycophenolate mofetil $50 \%$, plasmaferesis $37.5 \%$, hemodialysis $25 \%$ and in $12.5 \%$ cyclosporine. 4 children has or had progression of the disease, in spite of appropriate treatment (1 has constant progression of sinusitis, 2 has end-stage renal failure, 1 died because of alveolar haemorrhage).

\section{Conclusion}

Female predominance and clinical features of GPA diagnosed in children were similar to those described in adults. However, none of our patients had subglottic stenosis and only in 2 cases was observed saddle-nose. Although GPA was appropriate treated, progression was observed in $50 \%$ children.

\section{Disclosure of interest}

None declared.

\begin{abstract}
Authors' details
'2nd Department of Internal Medicine, Unit of Allergy and Clinical Immunology, Jagiellonian University, Medical College, Cracow, Poland. ${ }^{2}$ 2nd Department of Internal Medicine, University Hospital, Cracow, Poland. ${ }^{3}$ Pediatric Nephrology, University Children Hospital, Cracow, Poland. ${ }^{4}$ Pediatric Pulmonology and Allergology, University Children Hospital, Cracow, Poland. ${ }^{5}$ Pediatric Neurology, Rheumatology and Rehabilitation, St. Ludwik Children Hospital, Cracow, Poland.
\end{abstract}


- Convenient online submission

- Thorough peer review

- No space constraints or color figure charges

- Immediate publication on acceptance

- Inclusion in PubMed, CAS, Scopus and Google Scholar

- Research which is freely available for redistribution 